\title{
COOL BLEIBEN. \\ LIEBESDISKURSE IN DER LITERATUR DER GEGENWART. ÜBERLEGUNGEN ZU ROMANEN VON ANTJE RÁVIC STRUBEL UND UWE TIMM
}

\begin{abstract}
Staying Cool. Love Discourses in Contemporary German Literature (Antje Rávic Strubel and Uwe Timm)
\end{abstract}

Metaphors of "coldness" have increased dramatically in our present time, as evidenced by the three texts to be examined. The two novels Unter Schnee (2001) and Kältere Schichten der Luft (2007) by Antje Rávic Strubel focus on (or: are concerned with) lesbian relationships and the unfulfilled dreams through ties between the sexes. The novel Vogelweide (2013) by Uwe Timm runs through various heterosexual couple-constellations, for which the author utilizes the foil of Goethe's Affective Affinities. He is conscious of the fact that the traditional marriage has lost its status as a role model. All characters in the novel are searching for a new "language of love," but in their pursuit they relapse to those clichés, which they sought to escape. 'Heat' and 'coldness' turn out to be metaphors that have become obsolete a long time ago. They now produce dichotomies and narrow the love-discourse to meteorological sensibilities. The strong presence of sensations of coldness in all three novels nevertheless is still revealing. It can be read as a Symptom - to stay with the metaphors of the texts - for a 'cooling-down' of the relationship between the sexes.

KEYWORDS: Metaphors of coldness; Love Discourses; Contemporary Literature

\section{1.}

Kältemetaphoriken haben Konjunktur in der Gegenwart, zugleich haben sie eine lange Tradition in der Literatur- und Kulturgeschichte. „Das Wetter und die Gefühle“1

\footnotetext{
${ }^{1}$ Vgl. Gernot Böhme: Das Wetter und die Gefühle. Für eine Phänomenologie des Wetters. In: Kerstin Andermann, Undine Eberlein (Hrsg.): Gefühle als Atmosphären. Neue Phänomenologie und philosophische Emotionstheorie. Berlin 2011, S. 153-166.
} 
war schon immer ein bevorzugtes Feld für philosophische Spekulationen und „der Held und sein Wetter“2 ein spannendes Thema für literaturkritische Untersuchungen. Spätestens seit Goethes Werther (1774) wissen wir, dass ,heiß` und ,kalt` keine abstrakten meteorologischen Befunde sind, sondern auf die jeweiligen Befindlichkeiten im Gefühlshaushalt der Geschlechter verweisen. Dabei ist das Herz zur universalen Supermetapher in einem Liebesdiskurs avanciert, dessen ,Höhen' und ,Tiefen “ wie auf einem Barometer abgelesen werden können. Das flammende Herz hat sich weltweit zum vielvermarkteten Sinnbild der leidenschaftlichen Liebe entwickelt, es ist aber - wie das Märchen Das kalte Herz (1827) von Wilhelm Hauff zeigt - immer gefährdet, zu ,erkalten', aus welchen Gründen auch immer. In dem Text von Hauff ist es die Gier nach Geld, die den Helden dazu bringt, sein warmes, pochendes Herz gegen ein „Herz von Marmelstein“3 einzutauschen. Mit der Folge, dass nicht nur seine Empathie für fremde Menschen auf Null sinkt, sondern er auch keine Liebe mehr empfinden kann und schließlich zum Mörder seiner Frau wird. ${ }^{4}$

,Kälte“ und ,Wärme' sind zwar relative Größen, die Unterscheidung zwischen den beiden Zuständen ist aber lebensnotwendig für den Menschen, wie Alexander Kluge im Begleitheft zu seiner DVD Landschaften mit Eis und Schnee (2010) behauptet hat:

Wesentliche Eigenschaften, ohne welche die Menschheit nicht überlebt hätte, stammen aus der Eiszeit. So z. B. die für Warmblüter wichtige Unterscheidung zwischen heiß und kalt: Grundlage aller GEFÜHLE. Insofern kann man sagen, daß wir Menschen aus der Kälte stammen. Zugleich wird man aber beobachten können, daß Herzenskälte dauerhaft nicht zu ertragen ist. ${ }^{5}$

Mit dem Begriff „Herzenskälte“ bewegen sich Negt und Kluge in einem Metaphernfeld, das Manfred Schneider in seinem Buch Die erkaltete Herzensschrift (1986) vor allem in Hinsicht auf autobiographische Texte im 20. Jahrhundert untersucht hat. Unter den veränderten medialen Bedingungen hat sich die alte „Flammenschrift“6 der Liebe - um eine Metapher von Ingeborg Bachmann aufzugreifen - in eine kalte

\footnotetext{
${ }^{2}$ Vgl. Friedrich Christian Delius: Der Held und sein Wetter. Ein Kunstmittel und sein ideologischer Gebrauch im Roman des bürgerlichen Realismus. Mit einem Vorwort von Wolf Haas. Göttingen 2011 (zuerst 1971 erschienen).

${ }^{3}$ Wilhelm Hauff: Das kalte Herz. Zweite Abteilung. In: ders.: Sämtliche Werke in drei Bänden. Bd. 2. München 1970, S. 303-320, hier S. 306.

${ }^{4}$ Oskar Negt hat den Text in seinem Essay Kältestrom als „deutsches Geschichtsmärchen“ über eine „tiefgreifende Krise der menschlichen Lebensverhältnisse“ im Übergang von der Agrarwirtschaft zur Industrialisierung gedeutet. (Oskar Negt: Kältestrom. Göttingen 1994, S. 9. Vgl. auch Wolfgang Schmidbauer: Das kalte Herz. Von der Macht des Geldes und dem Verlust der Gefühle. Hamburg 2011).

${ }^{5}$ Alexander Kluge: Stroh im Eis. Geschichten. Begleitbuch zur DVD Wer sich traut, reißt die Kälte vom Pferd. Landschaften mit Eis und Schnee. Filme. Berlin 2010, S. 5.

${ }^{6}$ Ingeborg Bachmann: Der gute Gott von Manhattan. In: dies.: Werke 1: Gedichte. Hörspiele. Libretti. Übersetzungen. Hrsg. von Christine Koschel, Inge von Weidenbaum und Clemens Münster. München/Zürich 1993, S. 269-327, hier S. 316 (zuerst 1978 erschienen).
} 
maschinenartige Kommunikationsform verwandelt. Die heiße, leidenschaftliche Liebe, deren gesellschaftliche Entstehungsbedingungen von Niklas Luhmann in seinem Buch Liebe als Passion (1982) scharfsinnig analysiert worden sind, hat sich im virtuellen Zeitalter ${ }^{7}$ dramatisch abgekühlt. Cool ist das Modewort einer neuen Generation, coolness zu einer Überlebensstrategie in der postmodernen Welt geworden. Das Prinzip coolness ist nicht nur in der Literatur der Gegenwart präsent, es hat auch längst Einzug in Wissenschaft und Popkultur gehalten. ${ }^{8}$

\section{2.}

Im Folgenden möchte ich zunächst an zwei Texten von Antje Rávic Strubel, die bereits mit ihren Titeln auf den Kältediskurs der Gegenwart verweisen, den Zusammenhang zwischen äußeren und inneren Temperaturen im Gefühlshaushalt der Geschlechter untersuchen. In einem zweiten Schritt werde ich dann auf Uwe Timms Roman Vogelweide (2013) eingehen, in dem die Kältemetaphoriken weniger offensichtlich sind.

Der Roman Unter Schnee (2001) von Antje Rávic Strubel spielt in dem kleinen tschechischen Dorf Harrachov im Riesengebirge, das nach 1989 versucht, sich als Skigebiet für Touristen aus dem Westen interessant zu machen. ${ }^{9}$ Es gibt ein paar Skipisten und Loipen, einen Lift und eine bescheidene touristische Infrastruktur, wobei eine Sauna der Gipfel des Luxus ist. Zur Handlungszeit herrscht allerdings ein so starker Schneefall, dass der Lift aus Sicherheitsgründen abgestellt wird. Den wenigen Touristen bleibt nur der Gang in die Sauna, um ihren tristen Ferienquartieren zu entkommen. Die beiden Zitate, die dem Roman vorangestellt sind - „But the world was lost inside her" von Don DeLillo und „Wir sind von allem getrennt. Und alles Seiende ist unerreichbar" von Emile Cioran -, lassen bereits erahnen, dass diese Trostlosigkeit eine Entsprechung in den Verhältnissen der Figuren zueinander hat.

Ich konzentriere mich hier auf die Beziehung zwischen Vera und Evy, die in Erinnerung an ihre erste Begegnung im Schnee zwei Jahre zuvor - damals waren die beiden Frauen abseits der Piste ineinander gerast - einen gemeinsamen Skiurlaub in dem Ort machen. Weder die Sauna noch der Glühwein können die alten leidenschaftlichen Gefühle wieder herstellen. Als dann auch noch der Lift abgestellt wird,

\footnotetext{
${ }^{7}$ Vgl. Ulrike Vedder: Geschickte Liebe. Zur Mediengeschichte des Liebesdiskurses im Briefroman „Les Liaisons dangereuses" und in der Gegenwartsliteratur. Köln 2002.

${ }^{8} \mathrm{Zu}$ coolness als kulturellem Phänomen vgl. Ulf Poschardt: Cool. Hamburg 2000 sowie Judith Kohlenberger: The New Formula For Cool. Science, Technology, and the Popular in the American Imagination. Bielefeld 2015.

${ }^{9}$ Antje Rávic Strubel: Unter Schnee. Episodenroman. München 2001. Nach dieser Ausgabe werden die Zitate im Folgenden direkt im Text nachgewiesen.
} 
versucht Vera der Enge des gemeinsamen Appartements zu entfliehen. Sie verlässt das Haus im Schneetreiben und verirrt sich. Erst nach Stunden wird sie von einem Rettungstrupp in einem tiefen Schneeloch gefunden, in dem sie von einer Lawine eingeschlossen war.

Evy hat sich natürlich Sorgen um die Freundin gemacht, zugleich merkt man ihr eine gewisse Gereiztheit an, wie folgende Passage zeigt:

Wenn Vera so verschwindet wie heute morgen, sagt sie nie, wohin sie geht. Sie sagt nur, sie braucht Zeit für sich. Ihren Freiraum, wie sie das nennt. Schon zwei Tage mit mir allein sind ihr zuviel. Als gäbe es für sie nur zwei Aggregatzustände. Sobald es nicht siedendheiß geht, will sie weg. Dann läuft sie hinaus in den Schnee [...]. (S. 86)

An einer anderen Stelle, die sich allerdings nicht auf Evy und Vera bezieht, wird über die Liebe ebenfalls in meteorologischen Metaphern geredet: „Liebe? Das ist für dich doch nur wie 'n Scheißwetterbericht. Mal is'n Tief und mal scheint die Sonne.“ (S. 105)

Warum alle Beziehungen so trostlos sind, warum die Liebe plötzlich entflammt oder erkaltet und welche Erwartungen die einzelnen Figuren an das Leben überhaupt haben, bleibt in dem ,Episodenroman' offen. Andeutungsweise spielt die Autorin auf den Ost-West-Konflikt und auf gescheiterte persönliche Hoffnungen im Verhältnis der Geschlechter an. Für die ,klassische' Ehe gibt es im Roman nur abschreckende Beispiele, für das lesbische Paar Vera und Evy gibt es ebenfalls keine positive Zukunft. Auch der Internet-Chat, in dem sich Adina, die einzige Jugendliche, die noch in dem Dorf zu wohnen scheint, regelmäßig an ihren Traumort Rio phantasiert, kann die Tristesse nur zeitweilig aufheben. Die Erzählung von ihrer Begegnung mit den beiden „Spice Girls“ (S. 126), als die Adina die beiden Freundinnen wahrnimmt, und der Bericht über das Lawinenunglück und die dramatische Rettung Veras aus dem Schneeloch, wird von ihrem Chat-Partner immerhin als ,coole Geschichte" (S. 132) gelobt. Für Adina hingegen sind darin ihre ganzen unerfüllten Träume enthalten, an deren Entschlüsselung und Erfüllung jedoch niemand interessiert ist.

Der zweite Roman Kältere Schichten der Luft (2007), dem ebenfalls zwei Zitate vorangestellt sind, die diesmal sehr direkt auf die kaputten gesellschaftlichen Verhältnisse anspielen - das eine stammt von Lord Byron, das andere von D.H. Lawrence -, nimmt viele Motive des Vorgängertextes auf. ${ }^{10}$ Auch hier geht es um eine lesbische Beziehung und die unerfüllten Träume im Verhältnis der Geschlechter. Der Handlungsort ist jedoch ein anderer. Statt im verschneiten Tschechien befinden wir uns in einem Sommercamp in Schweden, wo eine Gruppe von Betreuer/innen den Kanuurlaub von Jugendlichen aus Deutschland zu organisieren versucht. Sehr

${ }^{10}$ Antje Rávic Strubel: Kältere Schichten der Luft. Roman. Frankfurt a. M. 2007. Nach dieser Ausgabe werden die Zitate im Folgenden direkt im Text nachgewiesen. 
schnell zeigt sich, dass die Atmosphäre von Missgunst und latenter Gewalt geprägt ist, in der die Liebesbeziehung zwischen der aus Halberstadt stammenden Anja und einer rätselhaften fremden Frau, die wie aus dem Nichts am See in der Nähe des Lagers auftaucht, keine Chance hat. Die 30-jährige Anja gehört wie auch die anderen Betreuer/innen zu den Verlierern im deutsch-deutschen Vereinigungsprozess. Mehrfach fällt die Bezeichnung „Loser“ im Text. Alle sind Langzeitarbeitslose, die sich ihre schlecht bezahlte Tätigkeit im Camp als Abenteuerurlaub schönreden, in Wirklichkeit aber ihren unhaltbaren häuslichen Verhältnissen entflohen sind und das Geld dringend brauchen, um über den nächsten Winter zu kommen.

Obwohl der Roman im Sommer spielt und es sogar einige heiße Tage gibt, überwiegen die Empfindungen der Kühle. Das Wasser ist kalt und die Nächte im Schlafsack ebenfalls. Die in leichte sommerliche Kleider gehüllte namenlose Frau wirkt in diesem Ambiente wie ein Fremdkörper, sie würde eher nach Italien als nach Schweden passen. Zwischen Anja und ihr entwickelt sich eine Beziehung, die irreale Züge annimmt. Anja versucht der Fremden einen Namen und eine Geschichte zu geben, während sie für die Frau Wiedergänger eines ehemaligen Geliebten zu sein scheint. Anja, deren Phantasien vom Fliegen und Schaukeln auf ihre unerfüllten sexuellen Wünsche verweisen, schlüpft immer mehr in die ihr von der Fremden zugewiesene Rolle des jungen Matrosen als Sinnbild homoerotischen Begehrens. Die Liebesbeziehung zwischen den beiden bleibt jedoch merkwürdig unterkühlt. An einigen Stellen scheint sich die Situation zwar aufzuheizen, wenn von einem ,glühenden“ Körper (S. 56) oder „heißen“ Handflächen (S. 99) die Rede ist. Die „Hitze“ (S. 74) bleibt am Ende aber nur eine Erinnerung an eine Zeit, die Anja zur Entdeckung des ,in ihrem Körper verborgenen' Jungen geführt hat, den die Fremde zum Vorschein gebracht hat. Der Roman endet mit einer Passage, die auf den Titel Bezug nimmt und in der Anja sich als „Er“ imaginiert:

Er weiß, daß diesen Tagen, in denen sie [die fremde Frau - I.S.] anwesend war, nichts folgen wird. Nichts außer einem langen Warten. Einem einzigen gleichförmigen Ton, der gemacht ist aus der Hitze, dem Flirren der Sonne über Staubfeldern und Erntemaschinen, aus Rissen in der heißen Erde und dem Wunsch, sich aufzulösen, hinein in die kälteren Schichten der Luft. (S. 189)

Wie im ersten Roman Unter Schnee fällt auch im zweiten Roman der Begriff cool zweimal, bezeichnenderweise jedoch nicht im Zusammenhang mit den beiden lesbischen Paaren. Weder Vera und Evy noch Anja und die rätselhafte Fremde sind cool oder wollen cool sein. Um coolness bemühen sich die anderen. Die vier Frauen sehnen sich nach der großen leidenschaftlichen Liebe, machen aber die Erfahrung, dass diese schnell erkaltet und die Phantasie der einzige sichere Ort ist, wo die Wünsche ausgelebt werden können. Hinzu kommt, dass gerade die Liebe zwischen den Frauen von der Umwelt abgelehnt wird, wie die Aufschrift „no gays“ (S. 64) zeigt, die Anja eines Morgens auf einem Fußball entdeckt und zu Recht auf sich bezieht. Erfolg im Lager haben ,Typen“ wie der betont virile Gruppenleiter, der als „cool guy“ (S. 150) 
von den Mädchen wie den Jungen gleichermaßen umschwärmt wird. Wie sexuell aufgeladen die Atmosphäre im Lager ist, zeigt der Vergewaltigungsversuch, den einer der Männer Anja gegenüber unternimmt. Am Schluss des Romans wird ihr klar, dass dieser Übergriff nicht ihr als Frau, sondern dem Jungen galt, den er in ihr zu erkennen glaubte.

\section{3.}

Der dritte Roman Vogelweide (2013), den ich ausgewählt habe, verrät den routinierten Erzähler, zu dem sich Uwe Timm seit seinem Debütroman Heißer Sommer (1974) in mehr als vierzig Jahren kontinuierlich entwickelt hat. ${ }^{11}$ Hier soll es weder um die vielfältigen und raffinierten intertextuellen Bezüge auf die Liebeslyrik des Mittelalters - auf die Namen Wolfram von Eschenbach und Walther von der Vogelweide wird im Text überdeutlich angespielt - noch um die Verweise auf Goethes Wahlverwandtschaften gehen, die die Folie des Romans von Timm bilden. All das wäre eine eigene Abhandlung wert. ${ }^{12}$ Ich möchte den Fokus vielmehr auf die Kältemetaphoriken lenken, die sich erst beim wiederholten Lesen in ihrer Bedeutsamkeit für den Text erschließen.

Vogelweide ist ein Roman, der wie die Wahlverwandtschaften über Ehe, Liebe und Begehren nachdenkt und sich - anders als die beiden Romane von Strubel - in traditionellen heterosexuellen Beziehungsstrukturen bewegt. Aber auch an diesen sind die gesellschaftlichen Entwicklungen nicht spurlos vorübergegangen. Die Partnersuche der jungen Generation hat sich auf Portale im Internet verlagert und die Forschung arbeitet eifrig daran, einen „Glücks-Algorithmus“ (S. 207) zu entwickeln, der Um- und Irrwege auf der Suche nach dem Partner oder der Partnerin vermeiden hilft.

Es geht im Roman um zwei Paare - die Männer befinden sich wie Goethes Protagonisten im ,besten' Alter, d. h. sie sind jenseits der 50, die beiden Frauen deutlich jünger. Eschenbach ist ein erfolgreicher Softwareentwickler und Ewald ein international beschäftigter Architekt, Anna, die Frau von Ewald, arbeitet als Kunstlehrerin, und Selma, die Freundin von Eschenbach, als Silberschmiedin. Die Begegnung zwischen Eschenbach und Anna löst eine Kettenreaktion aus. Ihre Affäre lässt die alten Beziehungen in die Brüche gehen. Anna verlässt sowohl ihren Ehemann Ewald als auch ihren Liebhaber Eschenbach und geht in die USA, wo sie als Galeristin erfolgreich wird. Ewald und Selma finden als neues Paar zueinander, während Eschenbach

${ }^{11}$ Uwe Timm: Vogelweide. Roman. Köln 2013. Nach dieser Ausgabe werden die Zitate im Folgenden direkt im Text nachgewiesen.

${ }^{12}$ Vgl. Sandra Kegel: Was kostet ein Kilo Begehren? In: Frankfurter Allgemeine Zeitung vom 16. August 2013. 
auch beruflich einen Absturz erleidet und schließlich Zuflucht auf der winzigen Insel Scharhörn findet, wo er als Vogelwart zur Ruhe zu kommen versucht. Doch als Anna nach mehr als sechs Jahren überraschend einen Besuch bei dem ehemaligen Geliebten ankündigt, wird Eschenbach in den Strudel seiner einstigen Gefühle wieder hineingerissen.

Erzählt wird der Roman aus der Erinnerung von Eschenbach. Die erste zufällige Begegnung mit Anna bei einem Vortrag über Stadtplanung ist ihm als ,glückhafter Augenblick" (S. 21) im Gedächtnis geblieben, das zweite Treffen findet bei einer Vernissage in einer Galerie statt, wo beiden das „coole Getue“ (S. 28) der Leute auf die Nerven geht und eine flüchtige Berührung von Annas Hand in Eschenbach das Gefühl auslöst, er habe einen „elektrischen Schlag bekommen“ (S. 29). Die dritte Erinnerung bezieht sich auf einen gemeinsamen Besuch der beiden Paare in einem chinesischen Restaurant, wo das Essen und der Alkohol nicht nur die Stimmung, sondern auch die Temperaturen steigen lässt, und Anna begeistert von einem Polareroberer erzählt:

\begin{abstract}
Anna begann, Wangen und Stirn glühten, von der Antarktis [...] zu erzählen. Sie lese gerade eine Biographie von Ernest Shackleton. Was für ein Mann! Der wollte 1916 die Antarktis durchqueren, die Zähigkeit, mit der er das alles betrieben habe, die ersten beiden Expeditionen, dann die Reise mit der Endurance, das Schiff bleibt im Packeis stecken, wird zerquetscht, die Mannschaft campiert auf einer großen Eisscholle [...]. In dieser Kälte, in dieser Ödnis, in dieser Einsamkeit - Anna glühte vom siebzigprozentigen Schnaps und vom Erzählen - memorierte Shackleton Gedichte von John Keats und Robert Browning. (S. 46f.)
\end{abstract}

Während der Ehemann den Wortstrom seiner Frau leicht genervt unterbricht und spöttisch bemerkt, dass er eigentlich „eifersüchtig auf diesen Ernest“ (S. 47) sein müsse, zitiert Eschenbach einige Zeilen aus The Waste Land von T.S. Eliot, einem düsterem Poem über Kälte und Einsamkeit. Er stellt damit eine Nähe zu Anna her, die ihrem illiteraten Ehemann verborgen bleibt. Diese Annäherung im Zeichen polarer Heroen und Texte lässt für die Zukunft nichts Gutes ahnen. Eschenbach ist kein Shackleton, obwohl er sich am Ende des Romans - allein auf seiner Insel - wie ein „Polarforscher" (S. 10) vorkommt, aber nicht als unbeugsamer Eroberer, sondern als einsamer Mann, dem seine verlorenen Kameraden „erfroren und schon im Eis begraben" (ebd.) als Geister erscheinen.

Die Kältemetaphern ziehen sich durch den ganzen Roman. So wie die polare Kälte tödlich sein kann, ist auch die übermäßige Hitze gefährlich. Eine der leidenschaftlichen Begegnungen mit Anna weckt bei Eschenbach bezeichnenderweise die Assoziation, „mit Benzin übergossen worden“ (S. 320) zu sein. Gesucht wird nach einem dritten Zustand, der nicht mit modischer coolness verwechselt werden darf, sondern auf das Ideal der ,ebenen“ Liebe verweist, von der Walther von der Vogelweide in seinen Minneliedern gesprochen hat. Diesen Zustand hat Eschenbach am Ende des Romans in der Sorge für die Dinge und die Tiere auf seiner, Vogelweide‘, 
einer winzigen abgelegenen Insel in der Nordsee, gefunden. Entsagung ist an die Stelle des Begehrens getreten. Damit stellt sich der Roman in gezielt altmodischer Weise in die Tradition der Wahlverwandtschaften und erweist sich zugleich als melancholischer Altersroman eines Autors.

Betont abgeklärt ist auch die Sprache des Romans. ${ }^{13}$ Nur Ewald, der betrogene Ehemann, verliert die Contenance, als er seinen Konkurrenten zur Rede stellt (vgl. S. 198), und Anna vergreift sich zweimal im Ausdruck, wenn sie von „Arschgesichtern“" (S. 28) und ,ficken“ (S. 320) spricht. Eschenbach hingegen versucht die Gefühle, die er für Anna empfindet, mit Begriffen wie Begehren, Begierde, Verlangen, Passion einzukreisen, die sich aber für ihn als ungenügend erweisen. Die „Sprache der Liebe", von der Roland Barthes gesprochen hat, hat sich in Timms Roman abgekühlt und ist zum Gegenstand sprachkritischer und erzähltheoretischer Überlegungen geworden. Die emotionslose, genaue Beschreibung, auf die Eschenbach in Brehms Tierleben in dem Artikel „Kampfläufer“ stößt, beeindruckt ihn so sehr, dass er daraus eine lange Passage zitiert (S. 159-162). Für sein eigenes Schreibprojekt, eine Untersuchung über das Begehren im digitalen Zeitalter (vgl. S. 92), für das er im Auftrag eines Meinungsforschungsinstitutes Materialien gesammelt hat, möchte er sich diese „glänzende Wissenschaftsprosa“ (S. 159) zum Vorbild nehmen.

Dieses genaue Hinsehen, das sich eine ebenso genaue Sprache sucht, die wiederum das Beobachten schärft, wäre für die Beschreibung unseres Verlangens vorbildlich, dachte er. (S. 162)

Eschenbach wird diese Abhandlung nicht schreiben, sondern sich damit begnügen, seine Umgebung zu beobachten, Daten zu sammeln und sorgfältig mit der Flora und Fauna auf seiner Insel umzugehen. Er ist kein „Kampfläufer“ aus Brehms Tierleben, sondern ein „Strandläufer“ (S. 139), der den Strand nach Treibgut absucht und dabei manchmal auf kleine Dinge stößt, die sein Herz erfreuen.

\section{4.}

Wenn man die Romane von Strubel und Timm vergleicht, fallen die Unterschiede deutlich auf. Sie hängen sicherlich damit zusammen, dass die beiden verschiedenen Generationen angehören und unterschiedliche Autorentypen repräsentieren. Der entscheidende Punkt scheint mir jedoch zu sein, dass sich Timm in Vogelweide auf der Folie der Wahlverwandtschaften an Problemen und heterosexuellen Beziehungsmustern abarbeitet, die - wie er selbst weiß - in der heutigen Zeit nicht mehr die allein verbindlichen Begehrensstrukturen sind. Als vielbelesener und geübter Autor kennt er die Liebescodes in- und auswendig und misstraut daher der ,Sprache

\footnotetext{
${ }^{13}$ Vgl. die abfällige Kritik von Volker Hage im Spiegel 33 (2013), S. 104f., in der er sich über den „Sex im Konjunktiv“ mokiert.
} 
der Liebe‘. Sein Protagonist Eschenbach gerät dennoch in einen Zustand, den er weder emotional noch sprachlich bewältigen kann. Durch die Leidenschaft zu Anna fühlt er sich wie „Von Sinnen“, er möchte zwar „cool bleiben“, allein bei dem Wort wird ihm allerdings ,ganz heiß“ (S. 153). Strubel dagegen kann für ihre Beziehungen zwischen Frauen in Unter Schnee oder für ihre „Transgender-Romanze“"14 in Kältere Schichten der Luft auf keine hochrangigen literarischen Muster zurückgreifen, sie muss die ,Sprache der Liebe' für die neuen verwirrenden Gefühle ihrer Protagonistinnen erst entwickeln. Dabei greift sie zum Teil auf romantische Klischees zurück, wenn sie die begehrte Frau als Märchenfee entwirft, oder sie orientiert sich an einer Sprache der Sexualität, die merkwürdig unangemessen wirkt.

Im Misstrauen gegenüber der Sprache bzw. in deren Ungenügen, die ,wahren“ Gefühle ausdrücken zu können, berühren sich die beiden Autor/innen. Timm reagiert darauf mit dem Rückzug auf die Beschreibung, er versetzt seinen Protagonisten auf eine einsame Insel, wo er „Tabellen der Vogelarten, Wetter, Temperatur, Windgeschwindigkeit“ (S. 299) anlegt. In der letzten Begegnung mit Anna erlebt er einen melancholischen Abschiedsmoment, den Timm Eschenbach sprachlich aufs Äußerste verknappen lässt: „Und plötzlich spürt er das Verlangen, nach Nähe, Haut, Fleisch, Wärme, Schweiß. Ja, in dir, denkt er, außer mir sein." (S. 331)

Diese Art der Erfüllung bleibt den Protagonistinnen in Strubels Romanen verwehrt. Die Autorin experimentiert mit unterschiedlichen Liebescodes, wohl wissend, dass diese nicht zu den Gefühlen der Frauen passen. Die Rio-Geschichten Adinas in Unter Schnee und die Geschichten um das verlassene Haus, die Anja sich in Kältere Schichten der Luft für sich und die Geliebte ausdenkt, verweisen darauf, dass Begehren am ehesten im Bereich der Phantasie ausgelebt werden kann. Mit den Wärmeund Kältemetaphoriken, die Timm und Strubel gleichsam als Gefühlsbarometer verwenden, um die Beziehungen zwischen ihren Protagonist/innen zu charakterisieren, greifen die Autor/innen auf bewährte erzählerische Mittel zurück. Die Beobachtung, dass dabei die ,Kälte‘ sowohl in emotionaler wie in meteorologischer Hinsicht dominiert, lässt erahnen, dass sich das Klima zwischen den Geschlechtern in dramatischer Weise abgekühlt hat.

\section{Literatur}

Bachmann, Ingeborg: Der gute Gott von Manhattan. In: dies.: Werke 1: Gedichte. Hörspiele. Libretti. Übersetzungen. Hrsg. von Christine Koschel, Inge von Weidenbaum und Clemens Münster. München/Zürich 1993, S. 269-327.

Böhme, Gernot: Das Wetter und die Gefühle. Für eine Phänomenologie des Wetters. In: Kerstin Andermann, Undine Eberlein (Hrsg.): Gefühle als Atmosphären. Neue Phänomenologie und philosophische Emotionstheorie. Berlin 2011, S. 153-166.

${ }^{14}$ Beatrix Langner: Sex im Kopf, im Wald und anderswo. In: Neue Zürcher Zeitung vom 17. März 2007. 
Delius, Friedrich Christian: Der Held und sein Wetter. Ein Kunstmittel und sein ideologischer Gebrauch im Roman des bürgerlichen Realismus. Mit einem Vorwort von Wolf Haas. Göttingen 2011.

Hauff, Wilhelm: Das kalte Herz. Zweite Abteilung. In: ders.: Sämtliche Werke in drei Bänden. Bd. 2. München 1970, S. 303-320.

Hage, Volker: ,Sex im Konjunktiv“. In: Der Spiegel 33 (2013), S. 104-105.

Kluge, Alexander: Stroh im Eis. Geschichten. Begleitbuch zur DVD Wer sich traut, reißt die Kälte vom Pferd. Landschaften mit Eis und Schnee. Filme. Berlin 2010.

Kegel, Sandra: Was kostet ein Kilo Begehren? In: Frankfurter Allgemeine Zeitung vom 16. August 2013.

Kohlenberger, Judith: The New Formula For Cool. Science, Technology, and the Popular in the American Imagination. Bielefeld 2015.

Langner, Beatrix: Sex im Kopf, im Wald und anderswo. In: Neue Zürcher Zeitung vom 17. März 2007.

Negt, Oskar: Kältestrom. Göttingen 1994.

Poschardt, Ulf: Cool. Hamburg 2000.

Schmidbauer, Wolfgang: Das kalte Herz. Von der Macht des Geldes und dem Verlust der Gefühle. Hamburg 2011.

Strubel, Antje Rávic: Unter Schnee. Episodenroman. München 2001.

Strubel, Antje Rávic: Kältere Schichten der Luft. Roman. Frankfurt a. M. 2007.

Timm, Uwe: Vogelweide. Roman. Köln 2013.

Vedder, Ulrike: Geschickte Liebe. Zur Mediengeschichte des Liebesdiskurses im Briefroman „Les Liaisons dangereuses “ und in der Gegenwartsliteratur. Köln 2002. 